\title{
Appendiceal Mucinous Neoplasm
}

\author{
Ashanti L. Franklin, MD, Carmen D. Coles, MD, Navin Changoor, MD, Tanya Gonzalez, MPH, \\ Edward E. Cornwell III, MD, Rhonda Burch-Smith, MD, Terrence M. Fullum, MD \\ Department of Surgery, Howard University College of Medicine, Washington, DC, USA \\ (Drs. Franklin, Changoor, Gonzalez, Cornwell, Fullum). \\ Department of Pathology, Howard University College of Medicine, Washington, DC, USA (Drs. Coles, Burch-Smith).
}

\begin{abstract}
Introduction: Appendiceal intussusception is an infrequent surgical disease that can present acutely, mimicking acute appendicitis, or chronically with intermittent right lower quadrant pain. Lead points are associated with appendiceal intussusceptions in slightly $>70 \%$ of cases. Common lead points are endometriosis, mucinous neoplasm, adenoma, adenocarcinoma, and carcinoid. Surgical therapy is often required to manage the intussusception. In cases of nonreducible appendiceal intussusceptions or those that have a lead point, an appendectomy, ileocecectomy, or right hemicolectomy.
\end{abstract}

Case Description: We report a case of appendiceal intussusception with an intraoperative finding of a cystic mass and pathologic diagnosis of low-grade mucinous neoplasm in a healthy 23 -year-old female patient. She underwent a laparoscopic ileocecectomy.

Discussion: Appendiceal mucinous cystadenoma requires long-term follow-up after resection.

Key Words: Appendiceal intussusception, Mucinous neoplasm.

Citation Franklin AL, Coles CD, Changoor N, Gonzalez T, Cornwell III EE, Burch-Smith R, Fullum TM. Appendiceal mucinous neoplasm. CRSLS e2014.00016. DOI 10.4293/CRSLS.2014.00016.

Copyright (C) 2014 SLS This is an open-access article distributed under the terms of the Creative Commons Attribution-Noncommercial-ShareAlike 3.0 Unported license, which permits unrestricted noncommercial use, distribution, and reproduction in any medium, provided the original author and source are credited.

Address correspondence to: Terrence M. Fullum, MD, Department of Surgery, Howard University College of Medicine, Howard University Hospital, 2041 Georgia Ave NW, Washington, DC 20060, USA. Telephone: (202) 865-1286, Fax: (202) 865-3063, E-mail: tfullum@howard.edu

\section{INTRODUCTION}

Appendiceal intussusception is a very rare pathologic disease with an incidence of $0.01 \% .{ }^{1}$ Intussusception of the appendix was first reported by McKidd in 1858. ${ }^{2}$ Appendiceal intussusception can clinically present acutely or chronically with acute symptomatology similar to acute appendicitis. The chronic presentation includes intermittent right lower quadrant pain, nausea, vomiting, and hematochezia. Common lead points include endometriosis, mucocele, adenoma, adenocarcinoma, and carcinoid. In adults, previous reports have shown that appendiceal intussusception can be reduced by colonoscopy provided that there is no lead point; however, definitive operative therapy is often required to properly manage the intussusception. ${ }^{2,3}$ Appendiceal intussusception may involve the colon, and in 1 case report involving an infant, the appendiceal intussusception led to total colonic intussusception with the appendix projecting from the anus. ${ }^{4}$
We present a case of appendiceal intussusception that was diagnosed intraoperatively with an incidental finding of low-grade mucinous cystadenoma, as well as a review of the literature.

\section{CASE REPORT}

A healthy 23-year-old female patient presented to the emergency department with a 16-hour history of abdominal pain. The pain was located in the right lower quadrant, was colicky in nature, and was graded as 10 of 10 in severity. The patient denied previous episodes of similar abdominal pain. The last bowel movement was passed the night before presentation, and the last flatus occurred the morning of presentation. The patient denied nausea, vomiting, diarrhea, constipation, and blood per the rectum. She had no significant medical, surgical, social, or family history. On physical examination, her vital signs were stable. She had mild tenderness in the right lower 

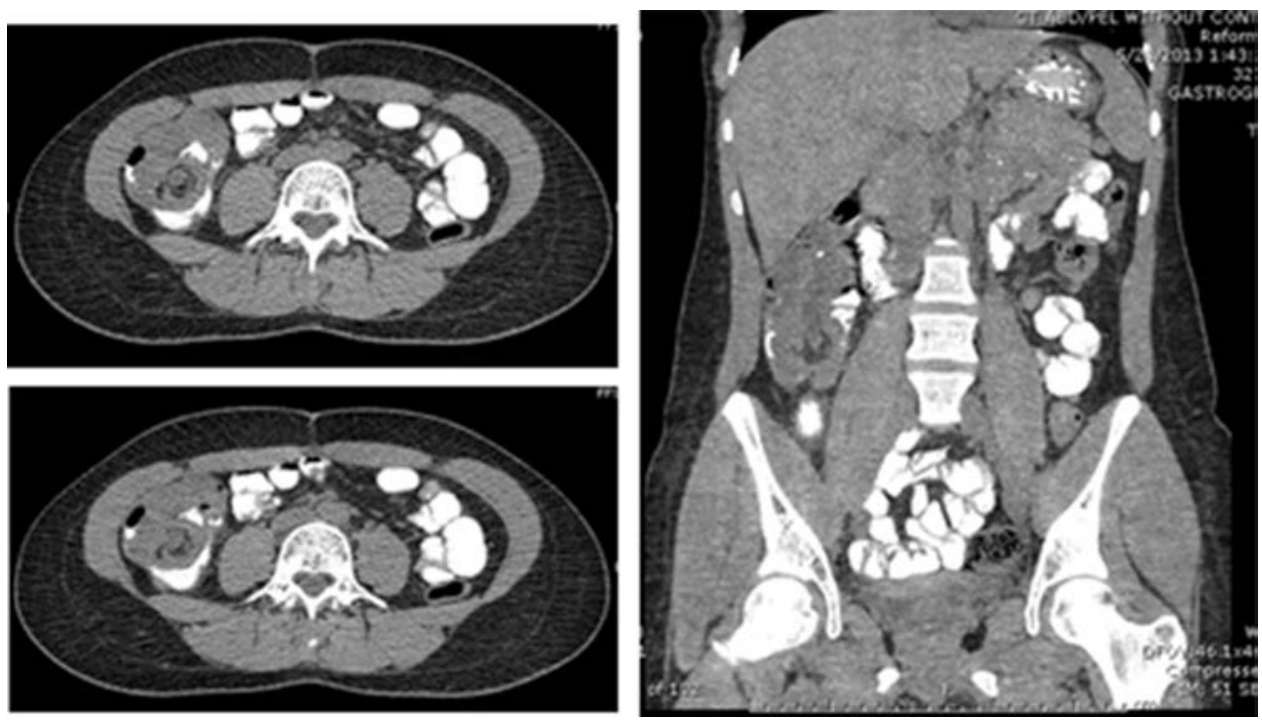

Figure 1. CT scan of ileocolonic intussusception.

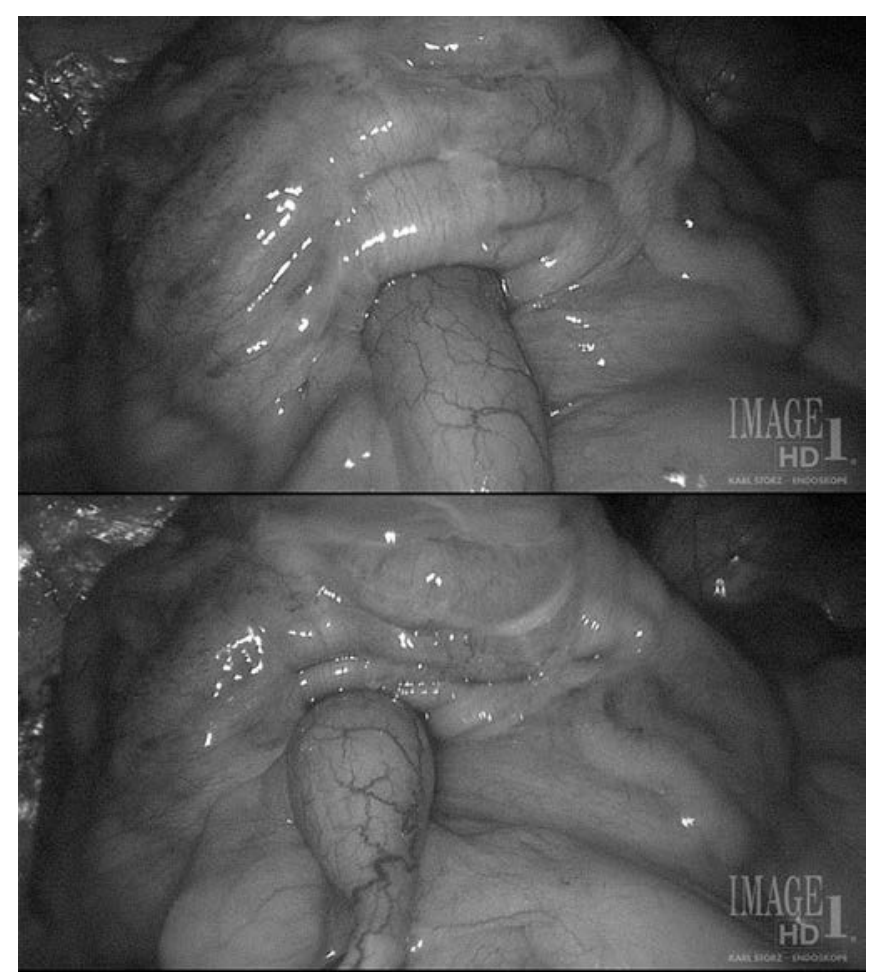

Figure 2. Intraoperative view of the appendix intussuscepting into the cecum, which was non-reducible intraoperatively.

quadrant, with slight tenderness to percussion in the right lower quadrant, and she had normal active bowel sounds. She had no guarding or rebound. Laboratory results showed a white cell count of $4.410^{9} \mathrm{~L}$ with no left shift. All other laboratory results were normal.
Computed tomography (CT) of the abdomen and pelvis with contrast showed an invagination of the distal small bowel into the ascending colon consistent with a large ileocolonic intussusception measuring $8 \mathrm{~cm}$ in length and $5 \mathrm{~cm}$ transversely and a small jejunojejunal intussusception (Figure 1). There was no lead point identified on CT; in addition, there was no evidence of bowel obstruction and no free fluid.

The patient was taken to the operating room for diagnostic laparoscopy. Intraoperatively, the base of the appendix was found to be intussuscepting into the cecum and encroaching on the ileocecal valve. Reduction of the intussusceptum was unsuccessful, and a laparoscopic ileocecectomy was performed with primary anastomosis of the ileum to the ascending colon (Figure 2). No jejunojejunal intussusception was noted. There were no other abnormal findings in the intra-abdominal cavity.

The pathology department received a segment of intestines including cecum $(3.9 \mathrm{~cm}$ in length $\times 9.5 \mathrm{~cm}$ in circumference), appendix $(5.6 \mathrm{~cm}$ in length $\times 1.5 \mathrm{~cm}$ in diameter), and terminal ileum $(4.0 \mathrm{~cm}$ in length $\times 3.8 \mathrm{~cm}$ in circumference). On gross examination, a large, yellowgreen mucous ball $(5.2 \times 4.0 \times 2.1 \mathrm{~cm})$ extruded from a dilated and bulbous appendiceal orifice into the lumen of the cecum (Figures 3, 4, and 5). The proximal one third of the appendix showed submucosal edema, and the remaining appendix was firm and fibrotic (Figure 6). There was no evidence of appendiceal rupture. The colonic mucosa surrounding the appendiceal orifice was erythematous and edematous but otherwise had no gross 


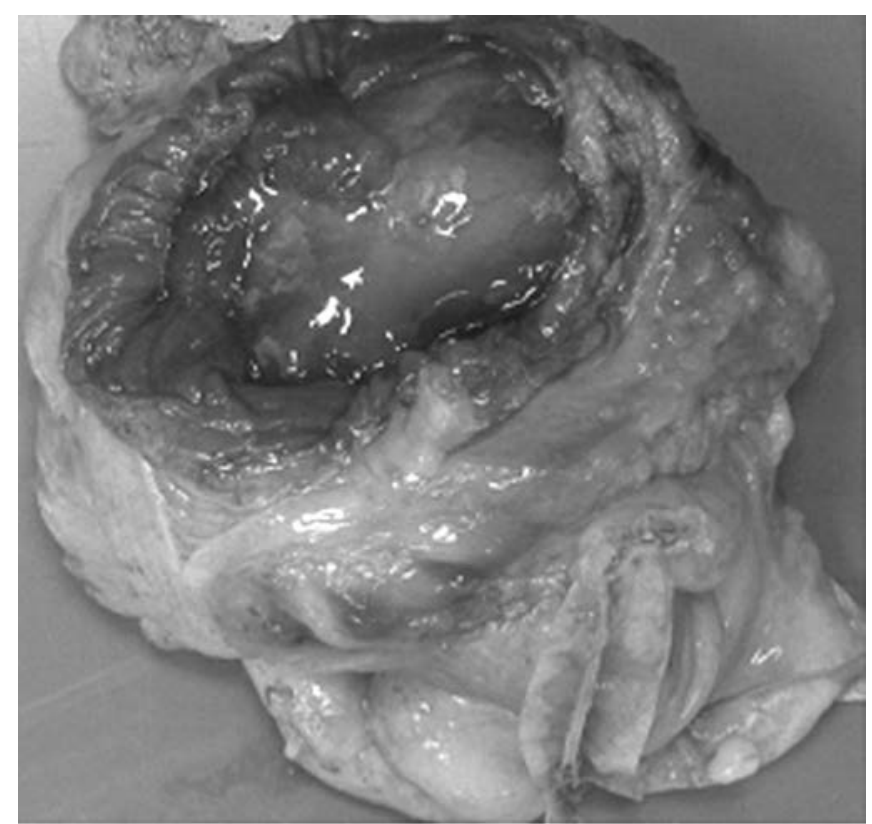

Figure 3. Segment of ileocecum (fresh). The tenacious mucus extruding from the postresection opening should be noted.

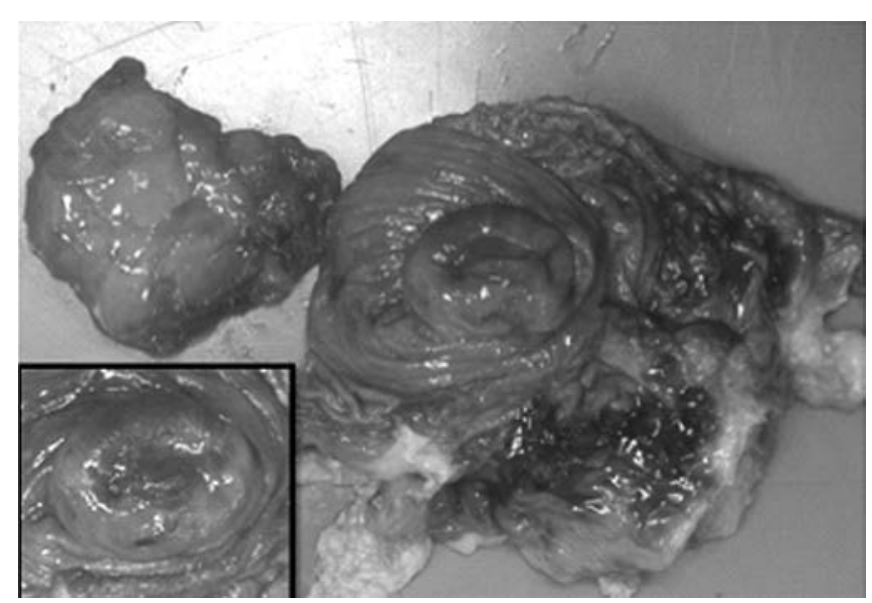

Figure 4. Segment of ileocecum (opened, fresh). Removal of the mucus shows a bulbous appendiceal orifice filled with similar mucus (inset). The remainder of the ileocecal mucosa is erythematous to hemorrhagic.

abnormalities. The portion of terminal ileum also showed no gross abnormalities.

On microscopic analysis, the appendiceal lumen contained large pools of acellular mucin with calcification. The mucosa was largely denuded; however, the preserved mucosa showed a single layer of well-differentiated hyperchromatic, pseudostratified cells with lowgrade dysplasia with areas of foreign body-type giant

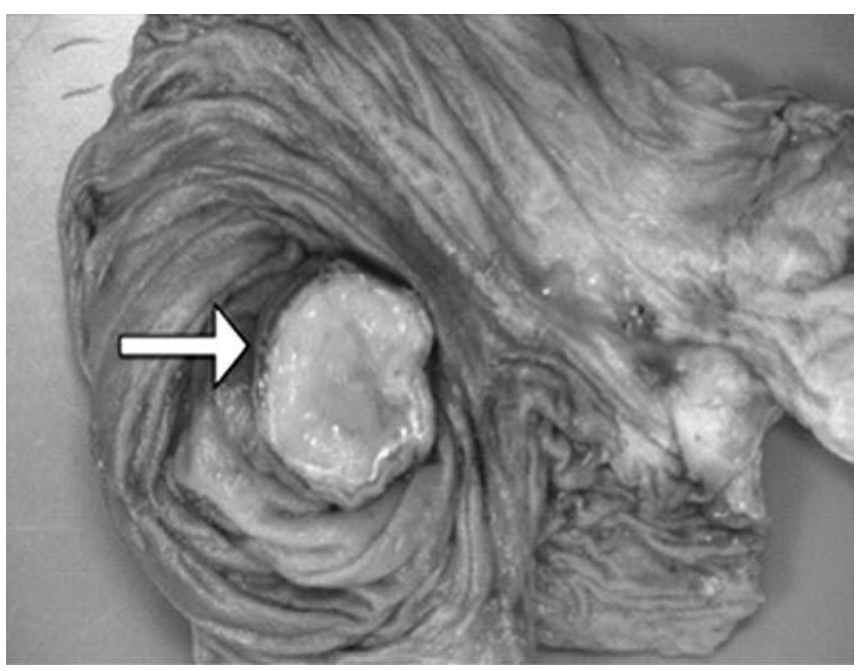

Figure 5. Appendiceal orifice (top sectioned) with formalinfixed tissue. There is marked dilatation of the appendiceal orifice (arrow), with a maximum diameter of $2.3 \mathrm{~cm}$, due to copious mucus.

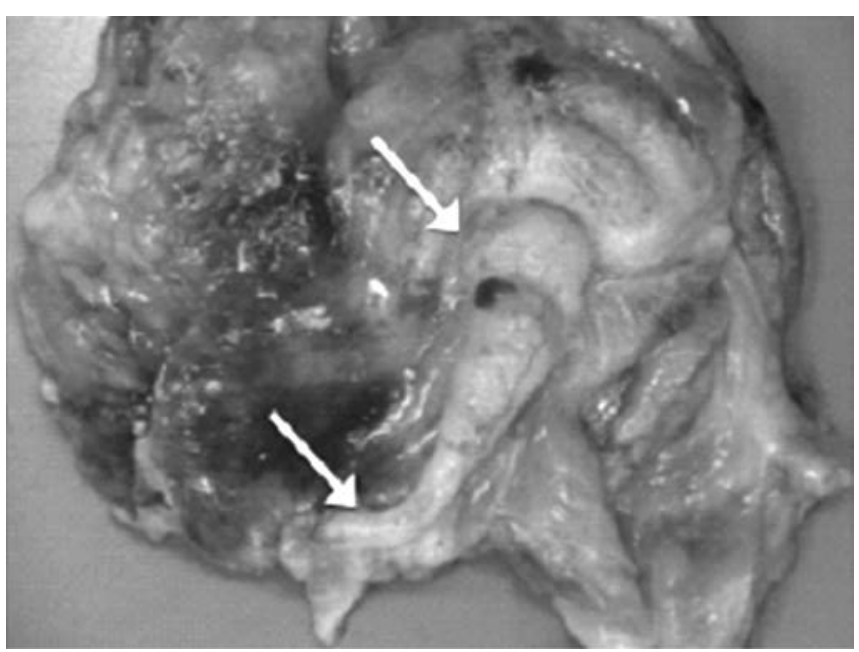

Figure 6. Vermiform appendix (fresh). One should note the dilatation of the proximal vermiform appendix (arrows) in comparison with the thinned fibrotic appearance of the distal aspect.

cells (Figures 7 and 8). The appendiceal wall showed no invasion by neoplastic cells. The pathologic results were consistent with a low-grade appendiceal mucinous neoplasm, the margins were uninvolved, and 3 lymph nodes were negative.

The patient's postoperative course was uneventful, and she had a return of bowel function, tolerated a mechanical soft diet, and was discharged home 3 days postoperatively. 


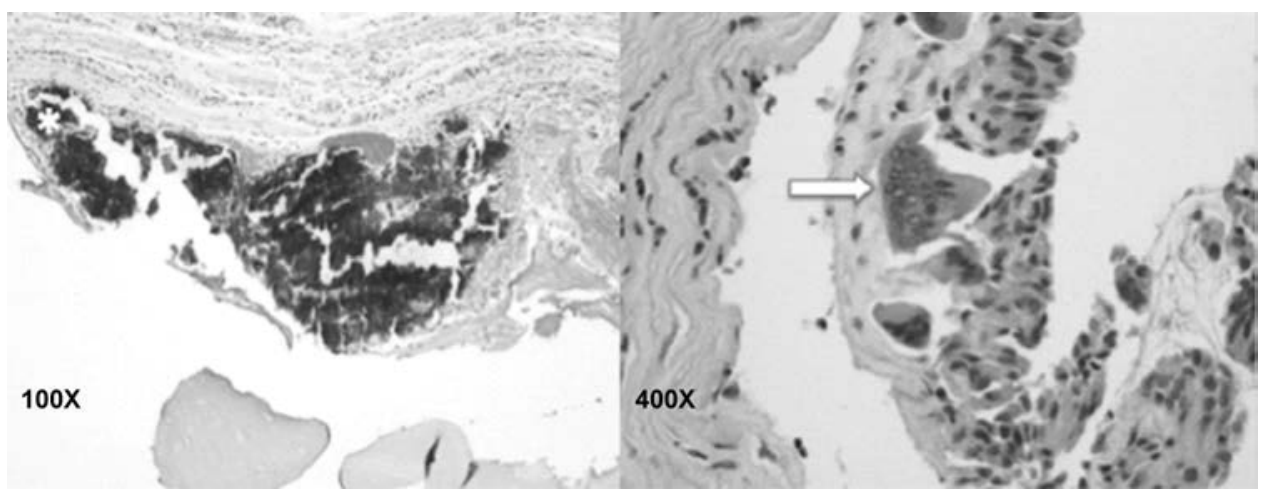

Figure 7. Vermiform appendix with hematoxylin-eosin staining. The appendiceal lumen contained pools of acellular mucin with calcification (star), as well as foreign body giant cells (arrow).
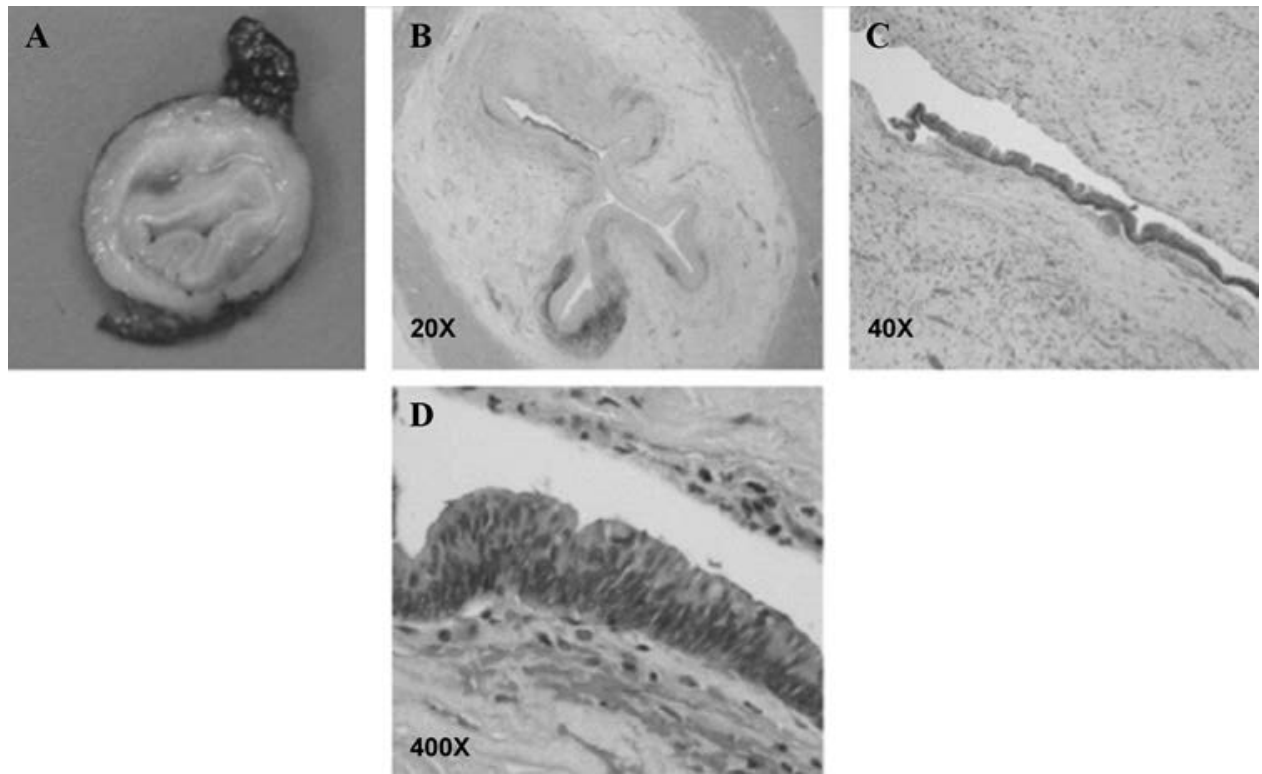

Figure 8. Cross section of vermiform appendix with hematoxylin-eosin staining. A, Gross cross section of proximal vermiform appendix. The increased thickness of the wall should be noted. B, Staining showing the increased thickness of the appendiceal wall due to fibrosis. In addition, there is loss of typical lymphoid aggregates within the appendix. C and D, Staining showing denudation of most of the epithelium and low-grade dysplasia with pseudostratified epithelium of the remaining mucosa.

\section{DISCUSSION}

Intussusception of the appendix is an infrequent pathologic condition. In a large autopsy series of $71000 \mathrm{pa}-$ tients, the incidence of appendiceal intussusception was $0.01 \%{ }^{1}$ It is more common in adults $(76 \%)$, with a preponderance in adult female to male patients of $2: 1^{2,3}$ Intussusception is defined as invagination of the bowel with its mesenteric fold (intussusceptum) into the lumen of adjacent bowel (intussuscipiens). According to McSwain ${ }^{5}$, appendiceal intussusception is anatomically classified by the part of appendix that is the intussusceptum.
McSwain described 5 types with varying degrees of appendiceal intussusceptum, from the tip of the appendix (type I) to complete invagination of the appendix into the cecum (type V), which is a progression of types I, II, and III. Type IV is a retrograde intussusception in which the proximal appendix invaginates into the distal appendix. ${ }^{2}$ Our patient had a McSwain type V nonreducible appendiceal intussusception that involved the entire appendix and the ileocecal valve.

The clinical presentation of appendiceal intussusception varies from asymptomatic to right lower quadrant pain 
mimicking appendicitis. Chronic symptomatology includes intermittent crampy right lower quadrant pain, vomiting, and intermittent lower gastrointestinal bleeding. Diagnosing appendiceal intussusceptions can be difficult, with more than half of the cases diagnosed at the time of surgery. Radiologic diagnosis includes ultrasonography in children with the pathognomonic "donut" or "target" sign. In adults, CT scan is the most commonly used method, showing a target-, sausage-, reniform-, or layered-type lesion in the cecum. ${ }^{6}$ If a mucinous appendiceal neoplasm is a lead point to the intussusception, CT may show a well-encapsulated mass with mural calcifications. ${ }^{7}$ Colonoscopy can be used to determine the etiology of appendiceal intussusception, as well as aid in reduction, provided that there is no lead point. 2,6

The pathophysiology of appendiceal intussusception involves abnormal appendiceal peristalsis caused by irritation and mobile mesoappendix with a wide appendiceal lumen or a thin mobile appendix. ${ }^{2,6}$ In the pediatric population this is due to inflammation of the appendix. In adults the most common lead point is endometriosis, carcinoid, mucocele, adenoma, or adenocarcinoma. In our patient the lead point was an appendiceal tumor. Fewer than 2\% of all surgically removed appendices are removed because of a primary appendiceal tumor. ${ }^{8,9}$ Of these, approximately $0.2 \%$ to $0.3 \%$ are removed because of an appendiceal mucocele. ${ }^{7}$ Low-grade appendiceal mucinous neoplasms occur predominantly in female patients, with the mean age being 50 years. Rarely, these cases occur in patients in their late teens. ${ }^{10,11}$ The clinical presentation of low-grade appendiceal mucinous neoplasm is usually right lower quadrant pain mimicking appendicitis. ${ }^{12}$

A pathologic diagnosis of low-grade appendiceal mucinous neoplasm remains controversial because there is no universally recognized classification system and there is wide variation in the terminology used. Depending on the institution, the lesion may be classified as an "adenoma," "cystadenoma," "mucocele," "mucinous tumor of uncertain malignant potential," or "low-grade mucinous appendiceal neoplasm." Generally, low-grade appendiceal mucinous neoplasms display undulating, villous or serrated architecture of the epithelium. However, the fibrous stroma that rests beneath the neoplastic epithelium distinguishes this neoplasm from an adenoma.9,13 In our case the low-grade dysplastic epithelium was confined to the appendix, with no evidence of invasion of the muscular wall by either the mucin or dysplastic epithelium. Thus a pathologic diagnosis of low-grade appendiceal mucinous neoplasm was warranted. In a Stanford University study, lesions with similarities in described histopathology to our case were considered "mucinous adenoma."10 In a Harvard University study, these types of lesions were considered a "low-grade appendiceal mucinous neoplasm" confined to the appendix. ${ }^{11}$ Despite the difference in nomenclature, the histologic findings were identical. Given the lack of a universal classification for appendiceal tumors, it is paramount that consistent terminology and communication of histologic findings are conveyed to all health care providers involved in the patient's care.

It is important to note that these low-grade tumors may be associated with pseudomyxoma peritonei (PP). In the Western population, the incidence of $\mathrm{PP}$ is 1 to 2 per million. ${ }^{12}$ The pathogenesis of $\mathrm{PP}$ is believed to be preoperative or intraoperative rupture of an appendiceal mucinous neoplasm with mucinous gravitational accretion into the intra-abdominal culs-de-sac, and $\mathrm{PP}$ represents a low-grade mucinous carcinomatosis. ${ }^{12,13}$ Perforation is not always seen, and PP may occur without invasion of the appendiceal wall. PP is relatively slow growing and is histologically classified by small islands of neoplastic epithelia. ${ }^{12}$ The behavior of PP is dependent on the cytologic atypia of the primary lesion (low grade vs high grade). ${ }^{13}$ Because of this, a thorough evaluation of the peritoneal cavity during surgical resection, as well as a thorough evaluation during follow-up by CT scan imaging, is critical. Once PP is diagnosed, surgical debulking is recommended; however, this remains controversial. Similarly, chemotherapy for PP also remains contentious.

Reducible appendiceal intussusceptions in low-risk populations are commonly treated with laparoscopic or open appendectomy. Some surgeons recommend an appendectomy with a cecal cuff because it reduces the risk of leaving an appendiceal stump and affords a margin of resection for any appendiceal lesion. ${ }^{2,14}$ Nonreducible appendiceal intussusceptions required ileocecectomy or right colectomy. In some cases of nonreducible appendiceal intussusceptions, surgeons have performed a cecotomy and resected the appendix. ${ }^{3}$ In our patient we performed a laparoscopic ileocecectomy with primary anastomosis. Studies have found that at 5 years' follow-up, low-grade appendiceal mucinous neoplasms rarely recur after initial resection. ${ }^{10,11}$ There is some controversy about performing a laparoscopic procedure for an appendiceal tumor, with concerns regarding adequate margins and decreased survival rates. ${ }^{15-18}$ In the presence of a ruptured appendiceal mucinous neoplasm or PP, conversion to open laparotomy should be considered. Long-term follow-up is recommended for patients with appendiceal neoplasms to evaluate for rare recurrences. 


\section{References:}

1. Collins DC. 71,000 human appendix specimens. A final report, summarizing forty years' study. Am J Proctol. 1963;14:265281.

2. Chaar CI, Wexelman B, Zuckerman K, Longo W. Intussusception of the appendix: comprehensive review of the literature. Am J Surg. 2009;198(1):122-128.

3. Park JK, Kwon TH, Kim HK, Park JB, Kim K, Suh JI. Adult intussusception caused by an appendiceal mucocele and reduced by colonoscopy. Clin Endosc. 2011;44(2):133-136.

4. McSwain B. Intussusception of the appendix. South Med J 1941;34:263-271

5. Dunavant $\mathrm{D}$, Wilson $\mathrm{H}$. Intussusception of the appendix, with complete inversion of the appendix and protrusion from the anus. Ann Surg. 1952;135(2):287-288.

6. Varsamis N, Pouggouras K, Salveridis N, et al. Appendiceal intussusception. In: Current Concepts in Colonic Disorders, Lule G, ed. ISBN: 978-953-307-957-8. InTech. Available at: http:// www.intechopen.comn/books/current-concepts-in-colonicdisorders/appendicealintussusception. 2012.

7. Bosman TF; WHOP classification of tumours of the digestive system. World Health Organizarion; International Agency for Research on Cancer, 4th ed. Lyon: IARC Press, 2010.

8. Adenocarcinoma of the Appendix. In: World Health Organization Classification of Tumors of the Digestive System. 8th ed. Sterling, VA: Stylus; 2008.

9. Panarelli NC, Yantiss RK. Mucinous neoplasms of the appendix and peritoneum. Arch Pathol Lab Med. 2011;135(10):1261-1268.
10. Pai RK, Beck AH, Norton JA, Longacre TA. Appendiceal mucinous neoplasms: clinicopathologic study of 116 cases with analysis of factors predicting recurrence. Am J Surg Pathol. 2009;33(10):1425-1439.

11. Misdraji J, Yantiss RK, Graeme-Cook FM, Balis UJ, Young RH. Appendiceal mucinous neoplasms: a clinicopathologic analysis of 107 cases. Am J Surg Pathol. 2003;27(8):10891103.

12. McDonald JR, O'Dwyer ST, Rout S, et al. Classification of and cytoreductive surgery for low-grade appendiceal mucinous neoplasms. Br J Surg. 2012;99(7):987-992.

13. Misdraji J. Appendiceal mucinous neoplasms: controversial issues. Arch Pathol Lab Med. 2010;134(6):864-870.

14. Day WA. Appendiceal intussusception simulating cecal tumor: case report. Dis Colon Rectum. 1963;6:118-120.

15. Gonzalez Moreno S, Shmookler BM, Sugarbaker PH, Appendiceal mucocele. Contraindication to laparoscopic appendectomy. Surg Endosc. 1998;12(9):1177-1179.

16. Bucher P, Mathe Z, Demirag A, Morel P. Appendix tumors in the era of laparoscopic appendectomy. Surg Endosc. 2004;18(7): 1063-1066.

17. Alexander R, Traverso P, Bolorunduro OB, et al. Profiling adult intussusception patients: comparing colonic versus enteric intussusception. Am J Surg. 2011;202(4):487-491.

18. Palanivelu C, Rangarajan M, John SJ, Senthilkumar K, Annapoorni S. Laparoscopic right hemicolectomy for mucocele due to a low-grade appendiceal mucinous neoplasm. JSLS. 2008; 12(2):194-197. 